\section{PHYSIOTHERAPY}

There is no necessity for the specialized groups of exercises which are required following thoracoplasty, and the simple post-thoracotomy regime alone is needed. As only two ribs are removed and the upper three left intact, there is no fear of developing scoliosis. Full scapula movement is obtained at the end of 10 days.

\section{Discussion}

Analysing the successful and unsuccessful cases, it is found that pneumonoplasty would be a suitable operation for controlling disease confined to the upper third of the lung. Cavities of up to $3 \mathrm{~cm}$. in diameter could be closed, but if they were of obvious tension type then obliteration was doubtful. If the disease extended right down to the hilum, then the perihilar region might not be controlled. Similarly gross disease involving the antero-lateral segment appeared to be difficult to deal with effectively. Bilateral apical disease, subject to the above limitations, would seem to be an ideal indication for this operation.

This operation has also been found useful in cases of apical disease where an upper lobectomy had been embarked upon but by reason of a densely adherent pleura no separation of the upper lobe could be obtained, and the alternative appeared to be an extrapleural pneumonectomy. To prevent loss of lower lobe function, a switchover to this type of operation after the exploration has been made is quite simple.

Figs. 2, 3, 4, and 5 demonstrate the results in a selected group of cases.

\section{SUMMARY}

Pneumonoplasty is an operation which aims at covering the relaxed upper part of the lung with a bony covering, and at the same time maintaining a relatively intact thoracic cage. It can be utilized in a considerable number of cases for which thoracoplasty has hitherto been employed and has the advantage of being a one-stage permanent collapse operation, with no visible deformity, and utilizing the body tissues only.

I have much pleasure in thanking for their help and suggestions in developing this operation Dr. John Gifford, of Fazakerley Sanatorium, Liverpool ; Dr. Clarke Penman, of Cheshire Joint Sanatorium; Mr. John Bickford, Mr. A. M. Mair, and the resident staff of the Liverpool Regional Thoracic Surgical Centre, Broadgreen Hospital, Liverpool.

\section{REFERENCE}

Bailey, C. P. (1941). J. thorac. Surg., 11, 326.

\title{
THE POST-OPERATIVE COURSE OF PNEUMONOPLASTY AND ITS MANAGEMENT
}

\author{
BY \\ J. HAMILTON GIFFORD \\ From the Fazakerley Sanatorium, Liverpool
}

The following notes are based primarily on 20 cases of pneumonoplasty in which $I$ have undertaken the post-operative treatment. In general it may be stated that the post-operative course is smoother than is usual following thoracoplasty and is attended by fewer complications both in variety and incidence, but there is an additional procedure of introducing air into the superior thoracic space for a short period,

\section{General Condition}

The general condition is surprisingly good when it is considered that the patient has undergone in one stage a lung mobilization approaching that of a sevenrib apical thoracoplasty. Post-operative shock may be rather more in evidence for two to three hours, but usually recovery is rapid so that after approximately eight hours the general condition is often better than that seen after the first stage of the latter operation.

Temperature and Pulse Rate.-The elevation of temperature is comparable to that following the first stage of a thoracoplasty, but a low grade pyrexia is frequently present for two weeks, and may persist longer. It is occasioned by the absorption of haemoglobin from the haemorrhagic fluid in the large superior thoracic space. There are rarely any symptoms of toxicity associated with it, and the patient is usually unaware of its presence.

The rise of pulse rate tends to be to a rather higher level than is usual after thoracoplasty. 
Dyspnoea.-As a rule dyspnoea is less marked owing to the absence of paradoxical respiration, but in the presence of excessive effusion in the superior thoracic space it may equal or exceed that experienced after thoracoplasty.

Dehydration.-This tends to be more in evidence due to the larger space into which fluid loss occurs, and more attention to fluid replacement is required for 48 hours.

Pain.-In the post-operative stage pain is generally much reduced, but on occasions is more severe than that following the first stage of a thoracoplasty. In its control morphine and codein are strictly avoided in order to prevent dulling of the cough reflex. Pethidine in doses of $50-100 \mathrm{mg}$. hypodermically is employed fairly liberally and is found to be effective. A mixture of A.P.C. (gr. 10) and phenobarbitone (gr. $\frac{1}{2}-1$ ) is often sufficient to procure rest.

Posture.-The kyphotic position, which is so often adopted and so frequently has to be corrected in thoracoplasty cases, is not encountered in pneumonoplasty patients, who therefore aerate the bases of their lungs much more effectively.

Coughing and Expectoration.-The comparative ease with which coughing is undertaken, and especially its effectiveness, are striking when first seen. Only in the occasional patient whose coughing is inhibited by pain, or apprehension of pain, is postural drainage required for perhaps 48 hours.

Surgical Emphysema.-During the first 36 hours some of the residual air in the superior thoracic space may be forced into the tissue planes of the neck by the increasing haemorrhagic effusion. The air is absorbed with the usual rapidity and causes little or no discomfort.

\section{Routine Management}

Wound.-In order to produce a fine scar alternate sutures are removed on the third slay, and the remainder on the sixth. On the latter occasion pieces of adhesive strapping are applied across the wound and left in situ until the tenth day.

Penicillin.-Penicillin (150,000 units intramuscularly) is given six-hourly for five to seven days as a routine.

Physiotherapy.-As only two ribs are resected in pneumonoplasty and the upper three are among those left intact, there is little tendency to scoliosis. Moreover the function of the anterior scalene is not interfered with and so the deviation of the head and neck so common after thoracoplasty is not seen.

There is thus no necessity for a full thoracoplasty regime, that employed after thoracotomy sufficing. In seven to ten days posture is normal and full range of movement of the arm, scapula, and spine regained. Thereafter the patient only requires to be put through this full range of movements daily, and exercises are directed solely to movements occasionally found to be a little weak or limited.

\section{AIR Refills}

Owing to the absorption of serum and effusion from the superior thoracic space which takes place between the seventh and fourteenth days there is a loss of support to the musculo-periosteal cupola over the collapsed lung, and the pressure in the space becomes sub-atmospheric. This allows coughing to produce gradual re-expansion of the lung and the pressure gradient tends to the same undesirable end. This was only fully realized after it had been seen to occur in some of the earlier cases. It was therefore decided to introduce air into the space until positive pressures were produced. Air refills are now given routinely beginning on the fourteenth day, and are continued at weekly intervals. Occasionally, when absorption of effusion is more rapid, the first three refills have to be given at three-day intervals, but thereafter weekly refills suffice.

There are three conditions which necessitate beginning refills earlier-namely, loss of fluid into the intrapleural space in cases where the pleura has been fenestrated at operation; when the fluid collection is small in amount from the outset due to little effusion occurring and/or early, rapid absorption; and finally when expectoration has been difficult and frequent, prolonged, and energetic coughing has been necessary.

Refills are never begun before the seventh day, because before this the superior thoracic space is still in direct connexion with the tissue planes of the neck, mediastinum, and chest wall. The introduction of air then results in surgical emphysema and its object is defeated ; indeed loss of some of the residual air from the space results.

The site chosen for the needle is in the first interspace anteriorly, though it is occasionally necessary to choose another owing to the presence of strands of fibrin.

Initially it is necessary to bubble air through the fluid which has collected in the space, as there is rarely a residual air-pocket which can be entered. If fluid is encountered with the needle used to administer the local anaesthetic (which should always be used until an air-pocket is established) it is well to employ a Record-fitting intramuscular needle for the refill and aspirate with a syringe sufficient fluid to ascertain that it is not venous blood that is being withdrawn. A Record-adapter fitted to the delivery tube from the pneumothorax apparatus then allows the air to be introduced without moving the needle.

It very seldom requires a pressure of over $15 \mathrm{~cm}$./ water to cause the air to bubble through the liquid, and if air does not enter when this pressure has been reached it is as well to remove the needle and repeat the initial procedure.

When an air-pocket has been found an ordinary Chandler refill needle is employed.

Once $20-30 \mathrm{ml}$. of air have entered, a manometric record is usually obtainable, but, if not, air is slowly introduced until the patient experiences a little tightness or sensation of weight in the upper chest. This amount is usually $100-200 \mathrm{ml}$. If a pressure record is 
obtainable the refill is stopped at $15 \mathrm{~cm}$./water, or earlier if these symptoms occur.

Subsequent refills are given to a pressure of $20 \mathrm{~cm}$./ water unless the patient experiences discomfort at a lower level. The amount of air required is of the order of $200 \mathrm{ml}$.

If surgical emphysema is produced at any stage, or if pain or discomfort supervenes, the introduction of air is stopped.

Should an increase of pyrexia occur following a refill, 250,000 units of penicillin in $10 \mathrm{ml}$. distilled water are injected into the space and repeated as deemed necessary.

Between the sixth and eighth weeks $x$-ray examination with a stationary grid will reveal recalcification taking place in the periosteum overlying the collapsed lung. The bony layer thus formed provides a sufficiently rigid support to the lung to prevent re-expansion so air refills are discontinued and the space allowed to fill up with effusion, from which the fibrin deposited eventually organizes.

After six weeks the patient can usually be allowed out of bed. The time spent out of bed and exercise are increased in such a way that he is fit for discharge to the follow-up clinic at the end of five months from the date of operation.

\section{Complications}

The following figures are based on the whole series of 37 cases.

Haemothorax.-This has been the commonest complication of note, occurring in six cases.

The type of case chosen for pneumonoplasty results in a high proportion having a visceral pleura which is only adherent locally at the apex, hence the parietal pleura which has to be stripped is often normal and easily fenestrated. When this occurs, in spite of the tear being sutured when possible, an intrapleural haemothorax which descends to the base results in the majority of cases. Owing to the presence of residual air in the superior thoracic space at the end of the operation the condition frequently becomes one of haemo-pneumothorax. The fenestration becomes sealed by fibrin deposition between 24 and 48 hours later, and being thus early it is only occasionally that the superior thoracic collection of fluid is seriously depleted.

The blood and secondary effusion have to be aspirated from the pleural sac at a site indicated by the physical signs and radiological appearances. At the same time as much air as possible is also removed. Usually three such aspirations suffice to reduce the effusion to a negligible amount which later becomes absorbed.

Systemic penicillin is given as part of the routine treatment during the period aspirations are most likely to be required, but, should they be necessary later, 250,000 units of penicillin in $10 \mathrm{ml}$. sterile distilled water are introduced into the pleural sac at the end of the procedure. All aspirations are conducted with full surgical aseptic precautions, including the use of sterile cap, mask, gown, and gloves ; all apparatus is autoclaved.

Excessive Effusion into the Superior Thoracic Space. -It may be mentioned here that in only one case was this due to reactionary haemorrhage. The collection of haemorrhagic liquid plays an important part in maintaining collapse of the lung in the initial stages and its amount should not be reduced unless definite signs of excess accumulation are present. Some feeling of tightness or heaviness in the upper chest on the operated side is common and in itself is not an indication for aspiration. But if there is marked tracheal displacement, bulging or bogginess of the supraclavicular fossa, pectoral region, axilla or interscapular area, sufficient fluid should be removed to reduce any tension in these areas and render the patient comfortable. This has been required in three cases.

The wound must be closely observed for signs of thinning and stretching, and, should these arise, it should be supported with padded elastoplast. In only two cases was this necessary.

Thrombophlebitis.-This complication may occur in the subclavian, axillary, and basilic veins, especially when there is excessive effusion present. It has been seen in two cases. The attention of the physiotherapist should be drawn to the need of assisting the venous circulation when excessive effusion arises. Anticoagulant therapy has not been employed in these cases, nor has it appeared to be necessary.

Pulmonary Complications.-There has been no instance of absorption collapse, acute embolic spread of disease, or acute exacerbation of existing disease in the post-operative stage.

Any patient with evidence of a fluid level in a cavity, or with a daily sputum volume of $1 \mathrm{oz}$. or more, is given pre-operative postural drainage on the morning of operation. In every case general anaesthesia has been employed, but during anaesthesia tracheal aspiration is carried out at intervals, especially when signs of secretion in the bronchi or trachea appear. At the end of the operation as a routine the larynx, trachea, and main bronchi are cleared by aspiration.

Infection.-Infection of the superior thoracic space has not occurred during the immediate post-operative stage, in spite of the introduction of air for a period of six to eight weeks.

Circulatory Failure or Collapse.-This has not been seen.

\section{SUMMARY}

The patient's general condition is an improvement on that following thoracoplasty, though the rise in pulse rate and dehydration may be a little more marked and a low-grade pyrexia rather more prolonged.

Only a post-thoracotomy regime of physiotherapy is required, and normal posture and movements are regained. Deformity is absent. 
On the third day alternate sutures are taken out. On the sixth day the remaining sutures are removed and adhesive strapping applied, and on the tenth day the adhesive strapping is taken off.

On the fourteenth day air refills are begun.

During the sixth to eighth weeks recalcification appears, the air refills are stopped, and the patient is allowed out of bed.
By the twentieth week the patient is up for eight to ten hours a day and is fit for discharge to the follow-up clinic.

I wish to record my gratitude to Mr. F. Ronald Edwards for his kindness in asking me to make this contribution to his account of pneumonoplasty and the assistance he has given me in writing it. I am indebted to both Mr. Edwards and Dr. O. F. Thomas for the privilege of managing the cases. 